This item was submitted to Loughborough's Research Repository by the author.

Items in Figshare are protected by copyright, with all rights reserved, unless otherwise indicated.

\title{
Using a design by features CAD system for process capability modelling
}

PLEASE CITE THE PUBLISHED VERSION

http://dx.doi.org/10.1016/0951-5240(94)90052-3

\section{PUBLISHER}

(c) Butterworth-Heinemann Ltd; Elsevier

\section{VERSION}

AM (Accepted Manuscript)

\section{LICENCE}

CC BY-NC-ND 4.0

\section{REPOSITORY RECORD}

Case, Keith. 2019. "Using a Design by Features CAD System for Process Capability Modelling”. figshare. https://hdl.handle.net/2134/13395. 
This item was submitted to Loughborough's Institutional Repository (https://dspace.lboro.ac.uk/) by the author and is made available under the following Creative Commons Licence conditions.

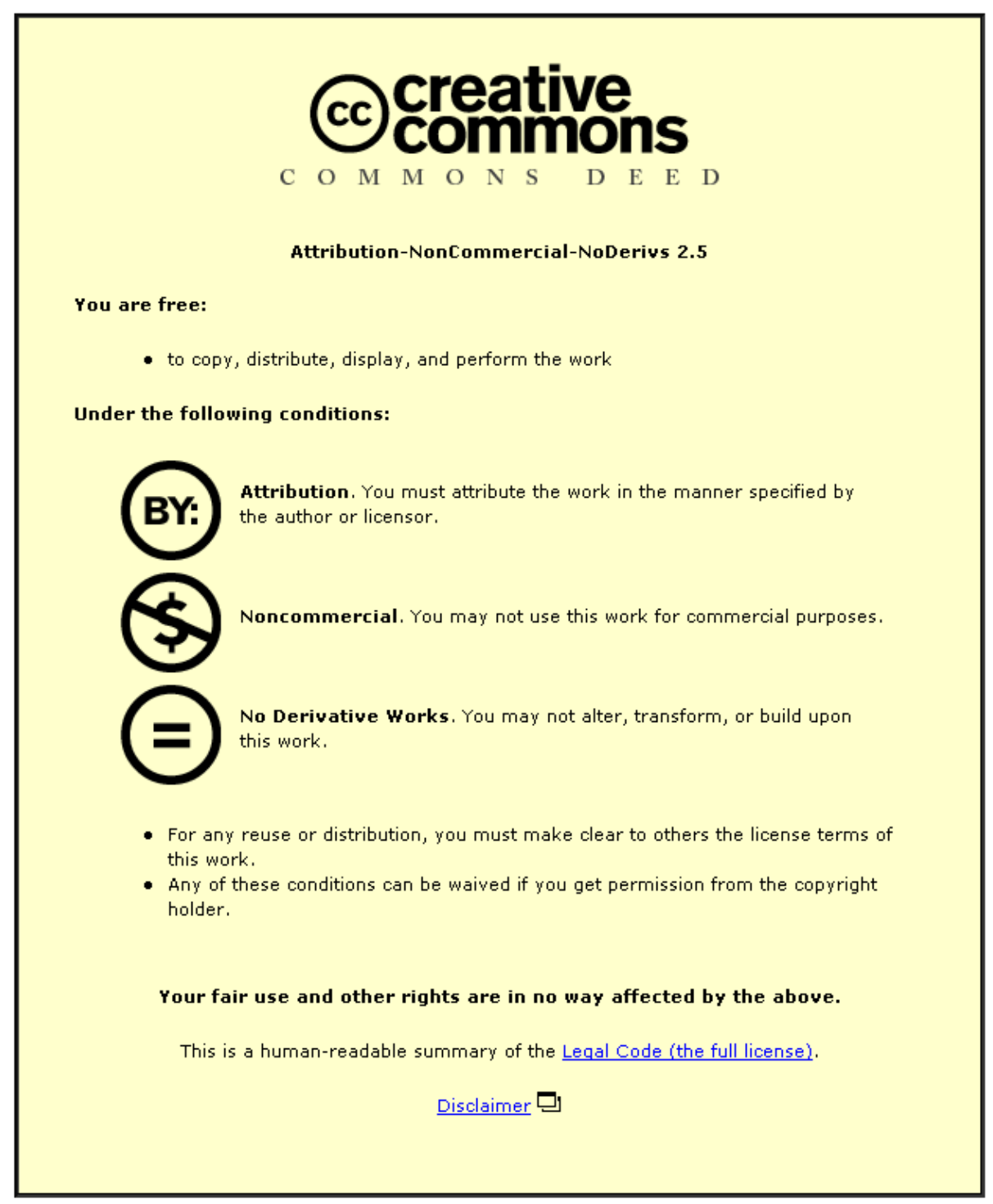

For the full text of this licence, please go to: http://creativecommons.org/licenses/by-nc-nd/2.5/ 


\title{
Using a design by features CAD system for process capability modelling Computer Integrated Manufacturing Systems Vol 7, No 1, February 1994, pp39-49
}

\author{
Keith Case \\ Department of Manufacturing Engineering, \\ Loughborough University of Technology, \\ Loughborough, Leicestershire LE11 3TU, UK
}

\begin{abstract}
Process capability modelling offers a method of matching the shape, technological and cost capabilities of manufacturing equipment to the requirements of components, singly or as groups. This provides the basis of planning tools useful in the capital intensive business of the construction of new manufacturing facilities or the reconfiguration of existing ones. The success of this modelling approach is dependent upon having an appropriate representation of the design geometry. The representation must be such that all geometric inquiries raised by the process capability modelling are either explicitly held within some data representation or alternatively can be derived algorithmically by reference to a geometric model. The representation must also be capable of withstanding the rigours of use within the wider context of implementing an important part of the CAM interface within a CIM environment. This paper describes a feature-based representation based on a feature taxonomy which uses External Access Directions (EAD) as the characterizing aspect of geometry. These EADs become potential machining directions for a collection of features on a component, and are used as an essential link into generative process planning activities. The representation has been used in conjunction with process planning and process capability modelling applications. This paper concentrates on the latter, where the feature representation has been embedded within a proprietary geometric modeller which has been provided with a purpose-built user interface. A feature-based component model is created by the geometric modeller and accessed by functions which enable flexible component grouping and matching to process capability through the concept of a composite component. Subsequent process component grouping within the context of particular manufacturing systems strategies (cellular manufacture, flow-line, etc.) ultimately results in functional machine descriptions and variants.
\end{abstract}

Keywords: feature-based design, process-capability modelling

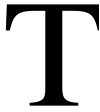

he pressures for integration of the many aspects of manufacturing planning arise from the increasing need to reduce product lead times in an environment where batch sizes are being progressively reduced in response to demands for customized products. Hence, whereas in the past the criteria for manufacture were frequently closely associated with optimization of machining times and maximization of the utilization of expensive manufacturing equipment, it is now more common in certain manufacturing situations to be more concerned with some overall 'product cost'. This total product cost cannot be simply measured in terms of material and machining costs in isolation from other aspects of the company's total set of activities. Instead, there is a need to assess the total 'life-cycle' implications of manufacturing decisions, and often this results in the placing of a 'benefit' on reduction of lead times that may well outweigh traditional cost considerations.

The obvious method of attempting to reduce total product development and manufacturing time is to employ computerized techniques to partially or totally automate the manufacturing planning activities. This is often described as a Computer Aided Engineering (CAE) approach. Extension of the idea to include similar methods for handling the operational aspects of actually producing products in some quantity (production planning and control) could then be described as the use of Computer Integrated Manufacturing (CIM) concepts ${ }^{1,2}$. Currently, most attempts to achieve this are overlaid on the traditional design-plan-manufacture sequence of activities. However, attempts to meet the objectives of CIM within a reduced total product lead time have more recently resulted in the application of concurrent (or simultaneous) engineering concepts, where appropriate activities in design and manufacturing planning progress in parallel rather than sequentially.

These objectives and the methodologies employed to achieve them are product centred and thus it is axiomatic that a satisfactory product representation must be at the heart of any computerized system which purports to provide this support. In the 1980s there were high expectations that the product representation inherent in the data structures of emerging solid modelling CAD systems were going to provide the necessary product description. This assumption was based on the undeniable fact that a properly defined geometric model within a solid modelling system has a complete knowledge of the geometry (within any restrictions inherently limiting the domain of the modeller). It would then seem to be a deterministic, if computationally intensive, activity to extract knowledge about geometry and use it for manufacturing planning purposes. However, this pleasingly straightforward scenario has been shown to be unrealistic in anything but the most simple situation. Solid modellers might be capable of simple but useful evaluations such as the determination of mass properties, but pure geometric modelling can only be considered as a starting point when it comes to the intellectually more demanding requirements of 
activities such as manufacturing planning.

The principal difficulty with traditional solid modellers is that their representation schemes are naturally preoccupied with geometric and topological aspects at a relatively low level, i.e. the underlying data structures are typically describing geometric entities such as vertices, edges and faces (boundary representation modellers) or primitives such as cuboids and cylinders (constructive solid geometry modellers ${ }^{3,4}$. For manufacturing planning purposes there is a need to have a higher level view where these geometric entities can be inter-related. For example, in process planning it is necessary to know that a collection of cylindrical holes are arranged in a particular pattern and can be accessed from a particular component direction. This activity of giving some higher meaning to collections of geometric entities is normally referred to as the use of 'Features' ${ }^{15}$. Features can thus be considered as a way of classifying particular sets of geometric entities into higher level representations that have some meaning for activities beyond those of geometric specification.

It is evident that the geometric entities of a component may be collected together into features in a variety of ways that reflect the needs of different design and manufacturing applications. Indeed, many recent research implementations of features show this domain or discipline dependency and it is common to talk of 'design features', 'functional features', 'manufacturing features', etc. For true integration of product life-cycle activities it is, however, very much preferable to have a single unified feature representation, or failing this a number of representations which can be readily mapped between each other.

The question then arises as to how a geometric representation is transformed into a feature representation. There are three possible alternatives. If computerized automation is not an objective, then human- assisted feature identification can be a useful technique. Here, a pure geometric model is interrogated by the (human) user and identified geometric entities are manually collected into groups and nominated as a feature. This has the single major advantage of not requiring a special purpose CAD system for its implementation, but the involvement of a human operator makes it an unsuitable contender as a methodology for computer automation. Replacing the human intelligence (in the grouping of geometric entities) by intelligent computer techniques overcomes this reservation. This approach is known as 'feature recognition' and is described in more detail elsewhere ${ }^{6-10}$. Feature recognition techniques could be said to emphasise rather than remove the traditional functional differences that exist in engineering design and manufacture, i.e. it takes as its starting point a geometric definition from the design phase and can have no knowledge of the decision-making process which lead up these conclusions. In a sense, feature recognition techniques have to 'second guess' the designer's intentions. If this could be achieved reliably and efficiently then this need not be a major barrier.
However, current feature recognition work does not usually exhibit these characteristics of efficiency and reliability. It is thus not surprising that a separate branch of features research has started from the premise of trying to capture the designer's intent and to express this in the form of features ${ }^{11,12}$. This design by features approach typically provides the designer with a library of features which not only have geometric meaning but could also have associated attributes that are meaningful to downstream manufacturing planning operations. Thus the designer might be able to specify that he requires a slot of given dimensional, positional and orientational parameters. This would be sufficient to create a geometric specification within a conventional solid modeller where a topological and geometric data structure is created to represent low level geometric entities. If the associations between these entities can be retained and supplied to manufacturing planning systems then we would consider this to be a design by feature approach.

The creation of a design by features system is conceptually simple, but in fact there are considerable difficulties. It is perhaps these difficulties which have prevented the commercial exploitation of the technique. There are some commercially available CAD systems which claim a features approach ${ }^{13,14}$, but in fact in these instances features are only used as a convenient method of describing geometry and have no impact on subsequent planning activities.

This paper describes a design by feature system conceived and implemented at Loughborough University as part of a wider research project aimed at providing methods for the strategic planning of major manufacturing facilities using process capability modelling techniques.

\section{Feature modelling}

Implementations of feature-based systems can be characterized according to a number of aspects:

- Underlying philosophy As described above, there are a number of alternative approaches to the use of features. The most important of these are feature recognition and design by feature. The former relies on intelligent software to interrogate the data structure of a geometric modelling system, while the latter creates a geometric data structure which is sympathetic to the needs of subsequent manufacturing planning activities. The two approaches are to a certain extent opposing schools of thought, but there is a growing realization among researchers that both methods have their merits and that hybrid systems might have much to offer.

- Geometric modeller Some feature-based systems are devoid of any geometric modeller (a geometric model is after all only a transitory representation as a means of communication between different design and manufacturing activities). However, most feature-based systems are built upon a geometric 
modeller. Most frequently these will be solid modellers, but in some instances two-dimensional modellers have been used (principally in activities such as turning where a $2 \mathrm{D}$ representation might be thought to be adequate). Solid modellers differ in their internal representations and thus boundary representation modellers are frequently used in feature recognition where their explicit, topologically-based data structures are suited to driving the interrogation algorithms. Constructive Solid Geometry (CSG) modellers have a superficial similarity with the feature primitives used in design by feature systems, and are thus frequently used in this context.

- Geometric domain Clearly, the kind of geometry that can be handled will be largely determined by the underlying geometric modeller. However, feature-based systems frequently use a sub-set of the total geometric domain, and particular systems are limited in their application. A common division is between prismatic and revolute parts, but this is a distinction that is disappearing as it is realized that modern machining cells are often capable of producing either type of component. Sheet metal work has also generated its own set of systems, partly because it is a simple geometric domain, but also because the bending and forming manufacturing processes used give rise to unexpected geometric complexities. Complexly curved surface objects cause difficulties for feature-based systems, largely because they can be featureless. There is some work which claims a features approach, but it could perhaps be more accurately defined as parametric design and manufacture.

- Feature taxonomies A principle objective of features is to enable the association of non-geometric parameters with geometric entities, and to infer extra information related to a manufacturing operation. Non-geometric information typically consists of such aspects as surface finish and tolerances, and in more sophisticated systems will include relationships with other features (parallelism, for example). Early features work concentrated on the enumeration of a wide variety of features without attempting to structure the information in any meaningful way. Really this was little more than parameterized design/ manufacture, and although useful in many practical manufacturing environments (where producing families of parts, for example), does not provide the generic solution required where high variety of product and small batch sizes are encountered. There are some analogies here with the alternative variant and generative approaches to process planning. A generative system is required for automation, and thus it is essential to be able to categorize all features into a limited and rigorous structure. Such a structure is known as a feature taxonomy ${ }^{15,16}$.

- Feature validation and editing Design is an iterative process where the final geometric form is the result of extensive creation, deletion and modification of the geometric specification. This causes feature recognition approaches no difficulty as they work off the final geometry. However, in a design by features approach it is necessary to control the changing of the feature model such that it holds valid information at the conclusion of the design phase ${ }^{17}$. In loosely defined feature taxonomies this may cause difficulties in recognizing when one feature changes into another.

\section{Feature-based design system (LUT-FBDS)}

A feature-based design system has been built on the principles described above, and forms an integral part of research into process capability modelling, i.e. the feature modeller is designed to provide information to a subsequent planning system which is capable of matching the geometric requirements of a component to the capability of a variety of (mainly) machining processes ${ }^{11}$. A typical feature-based component produced using the system is shown in Figure 1.

The methods of defining and manipulating components using features are generalized to suit any boundary representation modeller and the principal output on the design system is a component data model expressed in terms suitable for interpretation by any process planning or process capability modelling

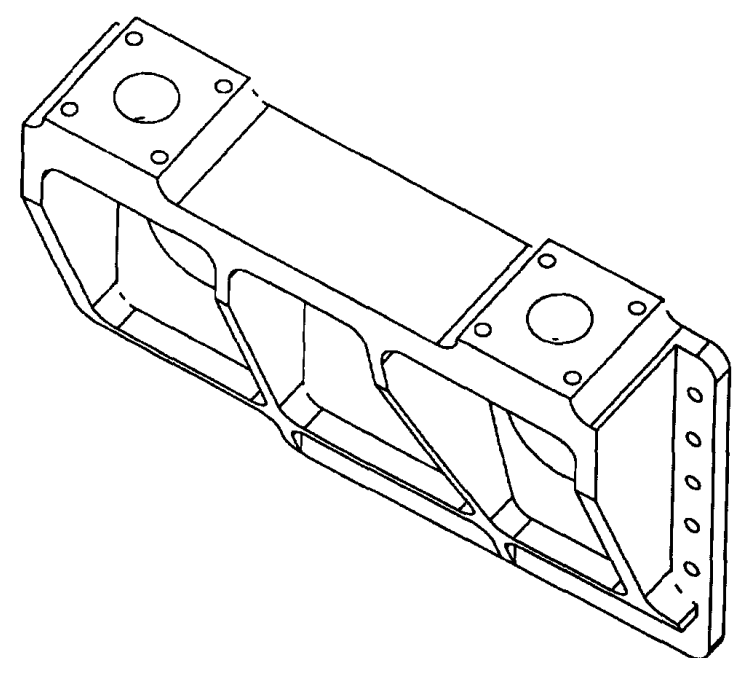

Figure 1. Typical component defined using features

demonstrate the principles is based upon Imaginer $^{1\lrcorner}$, a boundary representation solid modeller from Pafec and Horses $^{18}$, a User Interface Management (UIM) system also supplied by Pafec, but investigations are also underway to implement the system within the ACIS modeller ${ }^{19}$ using a Motif ${ }^{20}$ based interface. The architecture of the system is illustrated in Figure 2. 


\section{Design by features interface}

A major advantage of using established modellers is that it enables the use of most of the solid modeller's underlying capabilities whilst implementing our feature representation. Thus facilities for displaying and viewing the model, controlling the design database and accessing the data structure are handled by the solid modeller. The feature specific aspects, including a library of features with which to build a component model, editing facilities to modify existing features, means of defining relationships between features and feature validation are implemented to enhance or replace existing geometric facilities within the modeller. A typical example of the iconic features interface is shown as Figure 3.

The feature library is based upon a rigid feature taxonomy as shown in Figure 4. This taxonomy provides a precise topological description of all available features by reference to the faces of the features. Each feature has between zero and six imaginary faces plus any number of real faces. An imaginary face is an open region on the surface of the component (or another feature on the component) and will be created by the machining away of material. Real faces are those left on the component after machining. Figure 5 illustrates this for a simple slot feature which has three imaginary faces and a single real cylindrical face. Reference to the taxonomy of

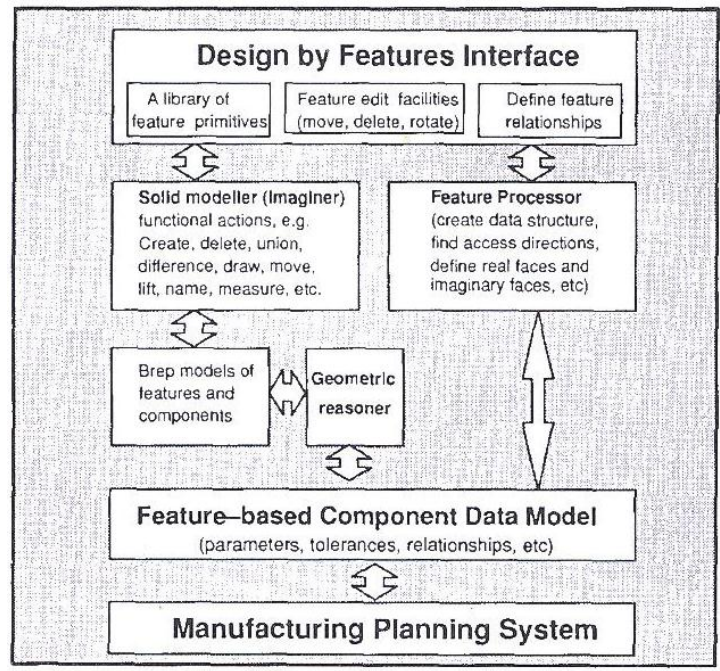

Figure 2. Architecture of the LUT-FBDS system

Figure 4 reveals that this is a depression (i.e. involves the removal of material), has three External Access Directions (EAD) and an open profile. The External Access Directions denote potential directions for machining and play a crucial role in subsequent process planning, i.e. they are an important determinant of the capabilities required of a manufacturing process and are useful in determining component set-ups. Figure 6 illustrates the External
Access Directions for each of the general classes of depression feature. The real faces on any particular feature are variable in number as they define an infinite variety of profile shapes. The taxonomy of Figure 4 is in fact extended to represent different profiles so that for example a through hole may be circular, square, trapezoidal, general shape, etc. The most useful of these profiles are provided through the icon interface, as illustrated in Figure 7. The extreme importance of using a rigourously defined taxonomy arises from the facility it provides to explicitly and uniquely characterize a nominated feature by reference to the topological structure held within the modeller's data structure. This is essential for subsequent manipulation of the component model through feature editing and validation. Figure 5 shows the structure for a slot feature where faces are named in a manner that explicitly describes the topology.

Facilities to edit features are provided in the form of commands to modify the dimensional, positional and orientational attributes. These are facilities that are very highly developed in commercially available solid modellers, and support the essential iterative nature of design activities. In the case of our feature modeller it is necessary to not only achieve this design flexibility, but also to record the effects of change within the data model. Figure 8 shows a fragment of a component data model and illustrates how these attributes are defined. Adequate maintenance of these attributes in relation to features is important for two reasons. Firstly, this information will form a major part of the design output to be passed onwards to manufacturing planning. Secondly, a representation that is capable of supporting feature validation is required, i.e. as mentioned earlier, changing these attributes could invalidate the feature through intentional or unintentional changes to feature class. Left unchecked this would generate a correct geometric model but provide misleading information to the manufacturing planning software. The strength of the approach adopted is that the precise and rigorous taxonomy provides us with a template against which features can be validated. Each feature has a specified number of imaginary faces, so that feature validation is concerned with comparing this requirement against the data structure of the solid modeller.

The final important aspect of the design by features interface is the ability to define feature relationships. When features are placed on a component it is necessary to define the way in which they relate to each other. Typical relationships that can be defined are ownership, grouping and tolerancing. Thus one 


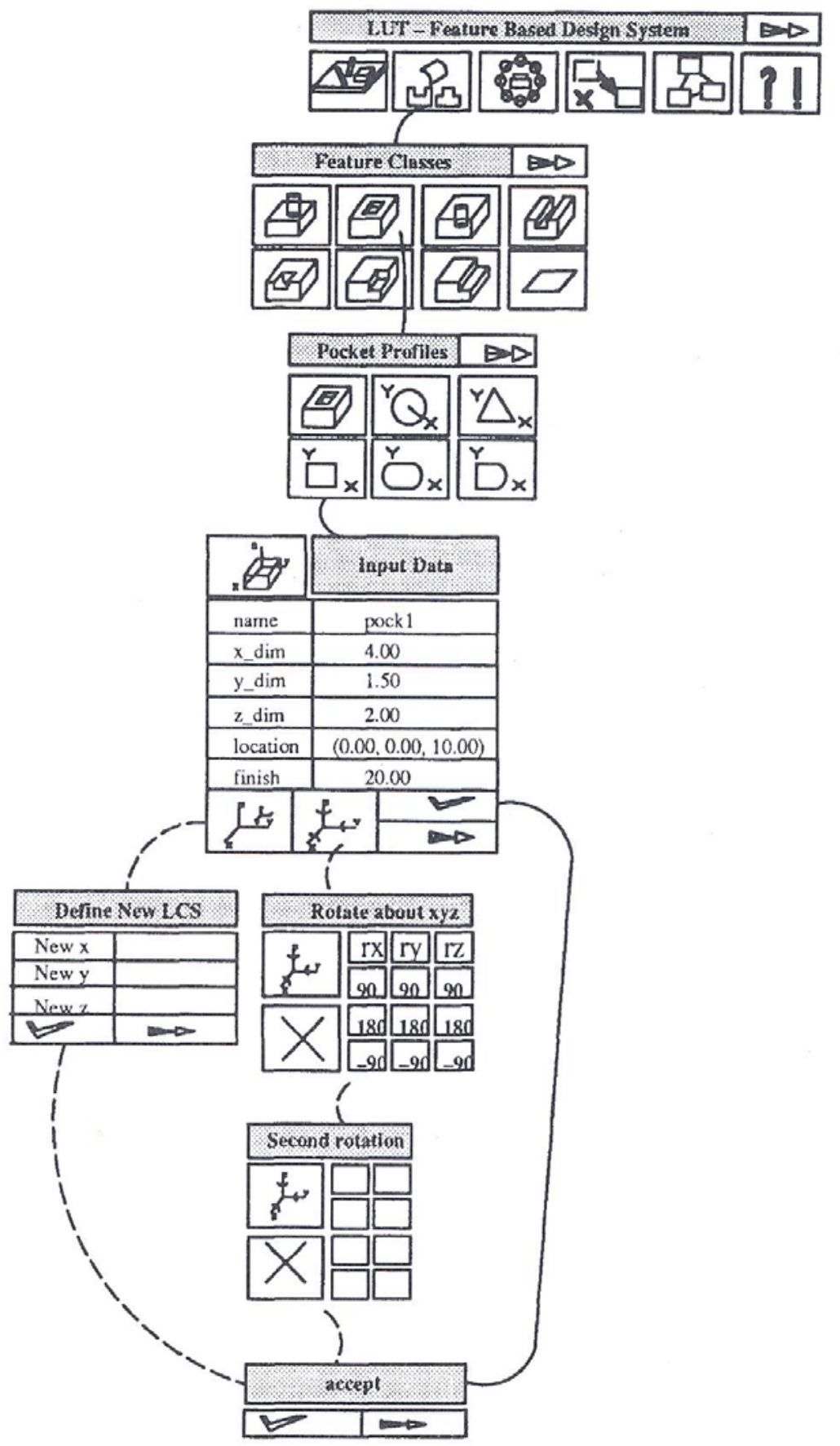

Figure 3. Example of the iconic user interface 


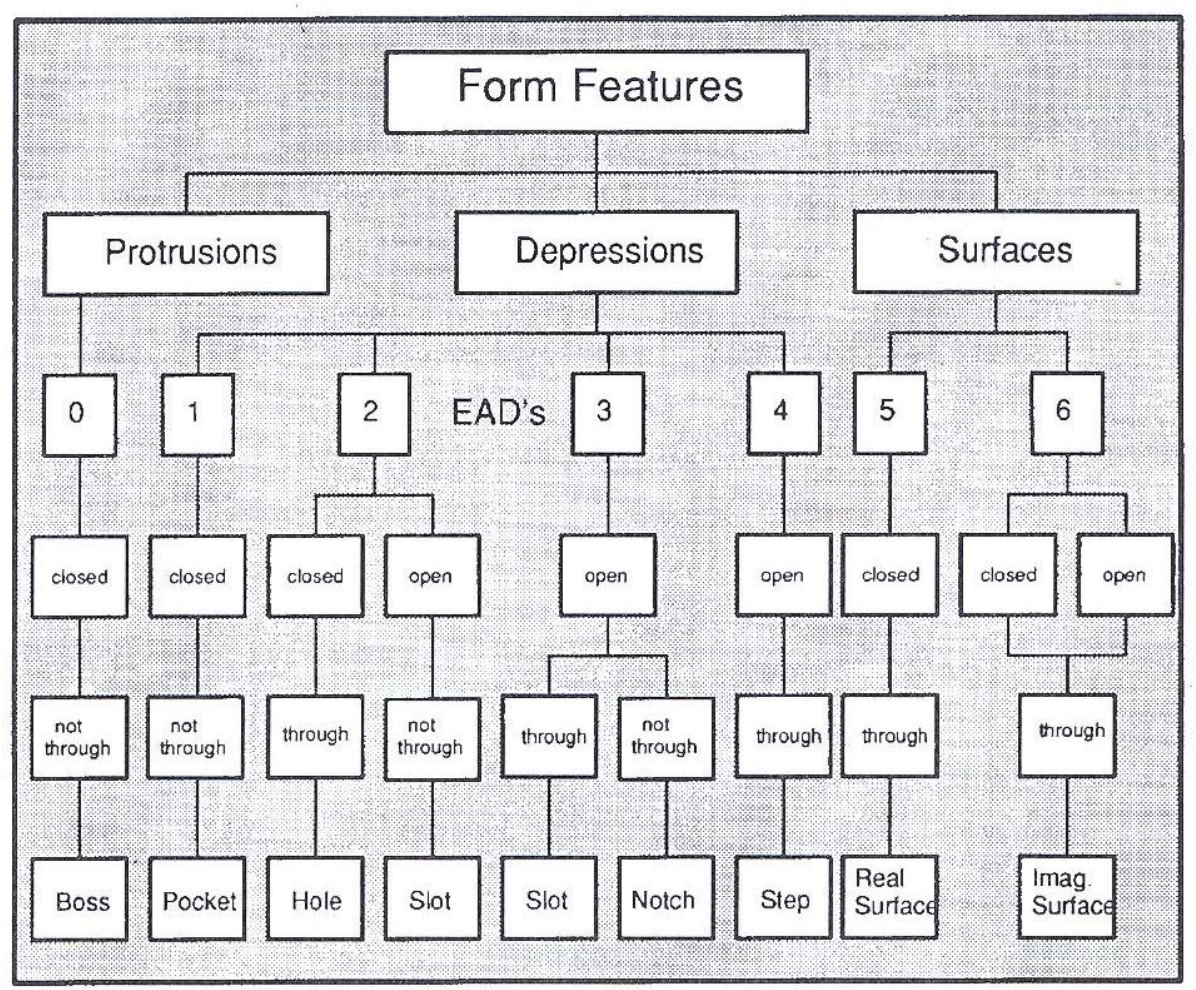

Figure 4. Feature taxonomy

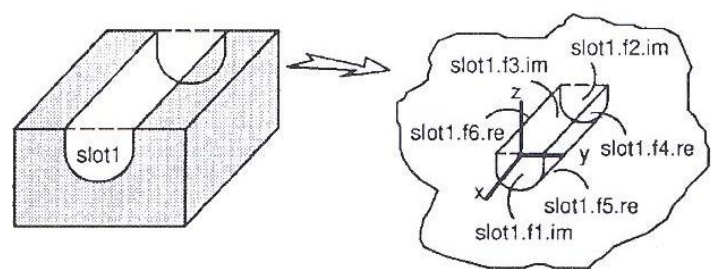

Figure 5. Topological representation of a slot

feature can be the 'child' of a 'parent', so that should the parent be moved then the child will be similarly affected. This would be used for example for a hole and its counter bore. Similarly, grouping relationships can be used to define 'compound' features such as holes on a pitch diameter. Tolerancing can be used to define functional/ manufacturing relationships such as parallelism between two or more features, and Figure 9 illustrates current capabilities in this respect.

\section{Feature processor}

The iconic interface provides a design interface of a type which has previously been shown to be beneficial $^{21}$. Preliminary experimentation with the interface has shown that it provides a more efficient and less error-prone method of specifying geometry than the underlying solid modeller. However, our aim is to capture this feature information for downstream use in manufacturing planning, and for this it is necessary to process the information into a suitable data structure. It may be preferable to redesign the solid modeller's data structure so that it can accommodate the extra information alongside its own

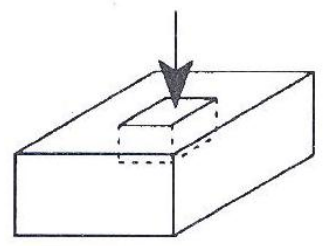

a) Non-Through Pocket: 1 EAD

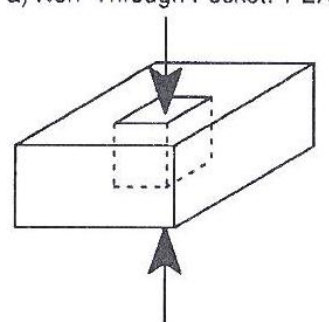

b) Through Pocket: 2 EAD's

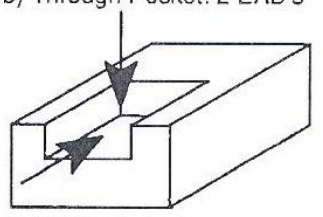

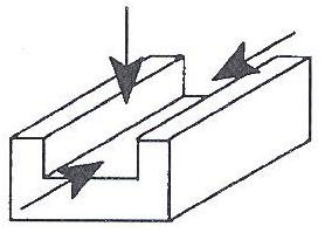

(d) Through Slot: 3 EAD's

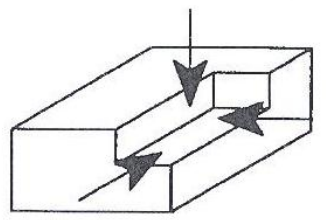

(e) Notch: 3 EAD's

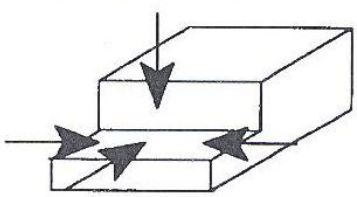

Figure 6. External Access Directions (EAD) for depression features

geometric data. However, this is likely to be difficult with a modeller that is aimed at many applications, and hence we have adopted the approach of maintaining a parallel data structure for the feature representation. 


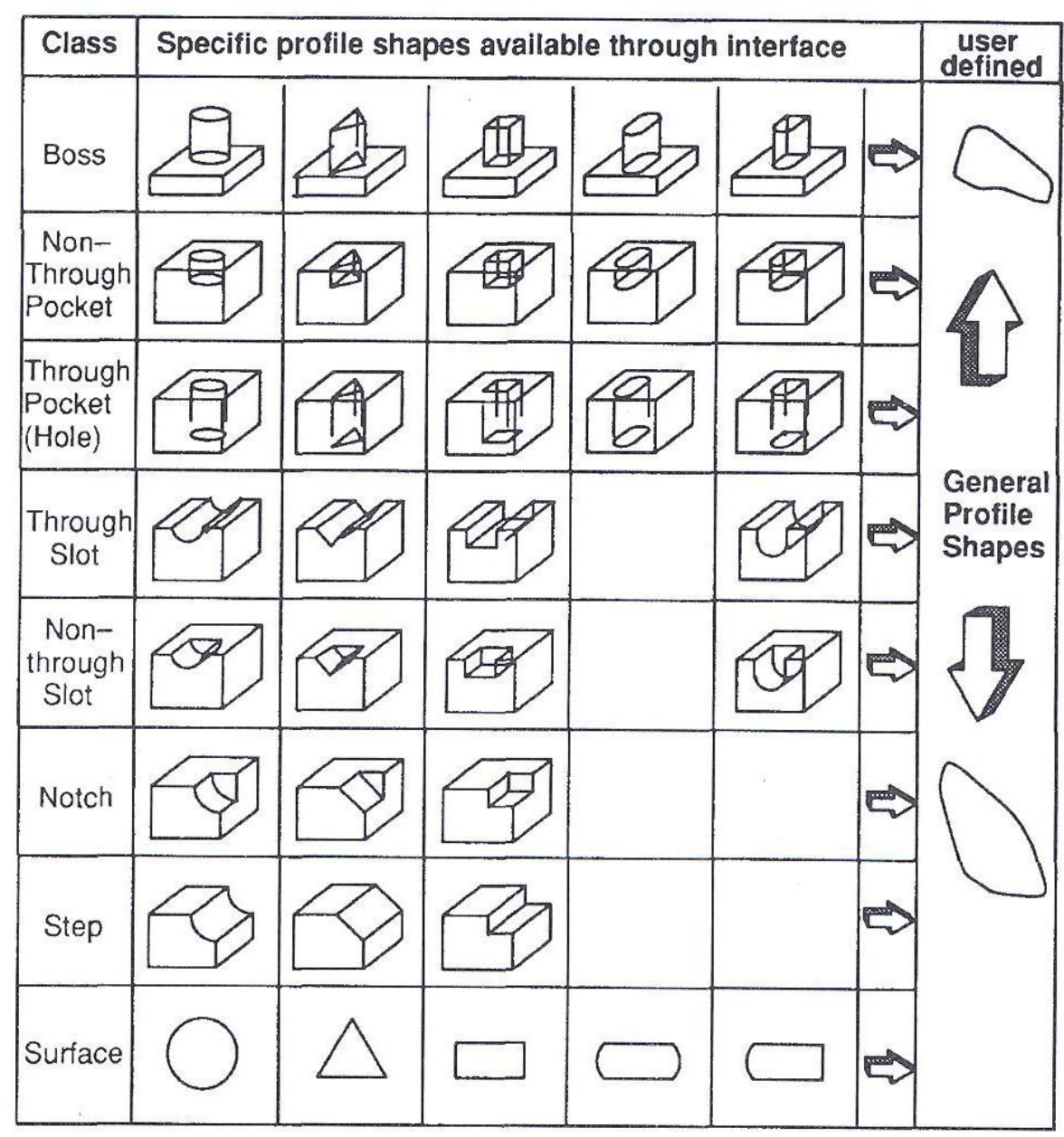

Figure 7. Library of feature primitives

Pointers between the two data structures provide the necessary links. Thus the feature processor handles the information provided interactively by the designer, and creates a data structure containing a knowledge of real and imaginary faces, feature relationships, access directions, etc.

\section{Geometric reasoner}

Manufacturing planning requires extra knowledge of the component geometry over and above that available in either the feature or geometric data structures. For example, the ability of a process to produce a feature will in part be determined by tool access. This is potentially dependent on the overall geometric condition of the component, and not only on the feature's own dimensions and attributes. Any attempt to manufacture the hole in Figure 9 from the direction of the pocket will need to know the clearance dimension within the pocket. The approach adopted towards solving these kinds of problems is the pragmatic one of pre-defining such geometric aspects as part of the feature data model.

\section{Feature-based component data model}

Figure 10 shows a simplified version of the feature-based component data model that provides the component description to the manufacturing planning software (and Figure 8 shows the information in more detail). The component is described in terms of a list of features, relationships and tolerances. The feature list contains sufficient detail to identify its position in the taxonomy, plus information relating to the individual faces of the feature and the feature attributes. This data model is created by the feature processor, and can be output in a number of forms including one which is suitable for entry into the Generis database that forms the basis of our planning system. The model is also seen as a natural point for the receipt of information from other CAD systems, perhaps expressed in some standard way such as by use of the STEP standard ${ }^{22,23}$. This would then provide a mechanism for integrating our planning system with alternative CAD systems.

\section{Manufacturing planning system}

The manufacturing planning system is described more fully elsewhere ${ }^{24}$, but has the objective of taking a 


\begin{tabular}{|c|c|c|}
\hline Component specifications & Tolerance definitions & Compound feature data \\
\hline $\begin{array}{l}\text { COMPONENT: exam plate } \\
\text { MATERIAL: cast_iron } \\
\text { STOCK ROUGHNESS: } 0.50 \\
\text { STOCK HARDNESS: } 120.00 \\
\text { NO OF FEATURES: } 7 \\
\text { NO OF TOLERANCES: } 2 \\
\text { NO OF RELATIONSHIPS: } 1 \\
\text { NO OF COMPOUND FTS: } 1 \\
\text { BLK SIZE: } \\
(100.00,140.00,40.00) \\
\text { BLK SURF FINISH: } 0.50\end{array}$ & $\begin{array}{l}\text { DATUM FT: pattern_h1 } \\
\text { DATUM ENTITY: axis } \\
\text { TOL FT: pattern_h2 } \\
\text { TOL ENTITY: axis } \\
\text { TOL TYPE: dimensional } \\
\text { TOL VALUE: } 0.10 \\
\text { DATUM FT: pattern_h1 } \\
\text { DATUM ENTITY: axis } \\
\text { TOL FT: pattern_h3 } \\
\text { TOL ENTITY: axis } \\
\text { TOL TYPE: dimensional } \\
\text { TOL VALUE: }-0.15\end{array}$ & $\begin{array}{l}\text { COMPOUND FT: pattern } \\
\text { FEATURE TYPE: hole4 } \\
\text { SINGLE FT1: pattern_h1 } \\
\text { SINGLE FT2: pattern_h2 } \\
\text { SINGLE FT3: pattern_h3 } \\
\text { SINGLE FT 4: pattern h4 } \\
\text { PARAMETER1: } 70.00 \\
\text { PARAMETER2: } 110.00 \\
\text { CENTRE: }(0.00,0.00,40.00) \\
\text { LCS X: }(1.00,0.00,0.00) \\
\text { LCS Y: }(0.00,1.00,0.00) \\
\text { LCSZ: }(0.00,0.00,1.00)\end{array}$ \\
\hline \multicolumn{3}{|l|}{ Parent-child relationship } \\
\hline $\begin{array}{l}\text { RELATION_ID: parent-child1 } \\
\text { PARENT FT: top_pock } \\
\text { PARENT FACE: top_pock.12.re } \\
\text { CHILDFT: bot hole } \\
\text { CHILD FACE: bot_hole.f1.im }\end{array}$ & & \\
\hline
\end{tabular}

\begin{tabular}{|c|c|c|}
\hline Basic parameters & Face list & Location, orientation \\
\hline $\begin{array}{l}\text { FT NAME: pattern_h1 } \\
\text { FT CLASS: pocket } \\
\text { SUB-TYPE: pocir } \\
\text { DIM1: } 0.00 \\
\text { DIM2: } 0.00 \\
\text { DEPTH: } 40.00 \\
\text { RADIUS: } 6.00 \\
\text { SURF FINISH: } 0.01\end{array}$ & $\begin{array}{l}\text { FT NAME: pattern_h1 } \\
\text { F1: pattern_h1.11.im } \\
\text { F2: pattern_h1.t2.re } \\
\text { F3: pattern_h1.13.re } \\
\text { F4: pattern_h1.14.re } \\
\text { F5: na } \\
\text { F6: na }\end{array}$ & $\begin{array}{l}\text { FT NAME: pattern_hi } \\
\text { LOCATION: } \\
\quad(35.00,55.00,40.00) \\
\text { LCS AXES: } \\
\text { X: }(1.00,0.00,0.00) \\
\text { Y: }(0.00,1.00,0.00) \\
\text { Z: }(0.00,0.00,1.00)\end{array}$ \\
\hline $\begin{array}{l}\text { FT NAME: top_pock } \\
\text { FT CLASS: pocket } \\
\text { SUB-TYPE: porec } \\
\text { DIM1: } 70.00 \\
\text { DIM2: } 80.00 \\
\text { DEPTH: } 10.00 \\
\text { RADIUS: } 0.00 \\
\text { SURF FINISH: } 0.05\end{array}$ & $\begin{array}{l}\text { FT NAME: top_pock } \\
\text { F1: top_pock.11.im } \\
\text { F2: top_pock.f2.re } \\
\text { F3: top_pock.13.re } \\
\text { F4: top_pock.14.re } \\
\text { F5: top_pock.15.re } \\
\text { F6: top_pock.16.re }\end{array}$ & $\begin{array}{l}\text { FT NAME: top_pock } \\
\text { LOCATION: } \\
\quad(0.00,0.00,40.00) \\
\text { LCS AXES: } \\
\text { X: }(1.00,0.00,0.00) \\
\text { Y: }(0.00,1.00,0.00) \\
Z:(0.00,0.00,1.00)\end{array}$ \\
\hline $\begin{array}{l}\text { FT NAME: bot hole } \\
\text { FT CLASS: hole } \\
\text { SUB-TYPE: hoobr } \\
\text { DIM } 1: 8.00 \\
\text { DIM2: } 0.00 \\
\text { DEPTH: } 30.00 \\
\text { RADIUS: } 8.00 \\
\text { SURF FINISH: } 0.01\end{array}$ & $\begin{array}{l}\text { FT NAME: bot_hole } \\
\text { F1: bot_hole.f1.im } \\
\text { F2: bot_hole.f2.im } \\
\text { F3: bot_hole.f3.re } \\
\text { F4: bot_hole.f4.re } \\
\text { F5: bot_hole.15.re } \\
\text { F6: bot_hole.f6.re }\end{array}$ & $\begin{array}{l}\text { FT NAME: bot_hole } \\
\text { LOCATION: } \\
\quad(0.00,0.00,30.00) \\
\text { LCS AXES: } \\
\text { X: }(1.00,0.00,0.00) \\
\text { Y: }(0.00,1.00,0.00) \\
Z:(0.00,0.00,1.00)\end{array}$ \\
\hline
\end{tabular}

Figure 8. Example of component and feature data models

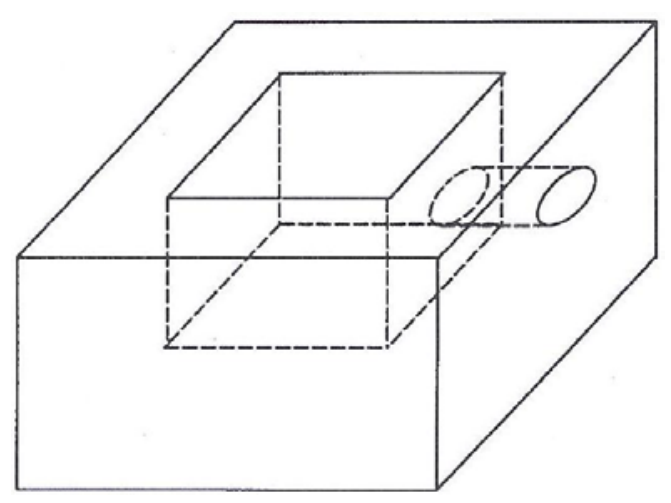

Figure 9. Accessibility requiring geometric reasoning sympathetically defined component data model (i.e. one that has been designed for this purpose) and using it in either process planning for manufacture or in the longer term strategic planning of manufacturing facilities. In either case, the problem is to match the shape, tolerance and relationship requirements expressed by the component model to available manufacturing facilities.

The capability of several metal cutting processes have been represented using a system of formative and positioning motions and cutting tool types. A series of transformations to link the process coordinate frame to the feature, component and machine tool coordinate frames have been developed. We are currently investigating a process taxonomy for description of the processes by a set of attributes linked to the volumes 


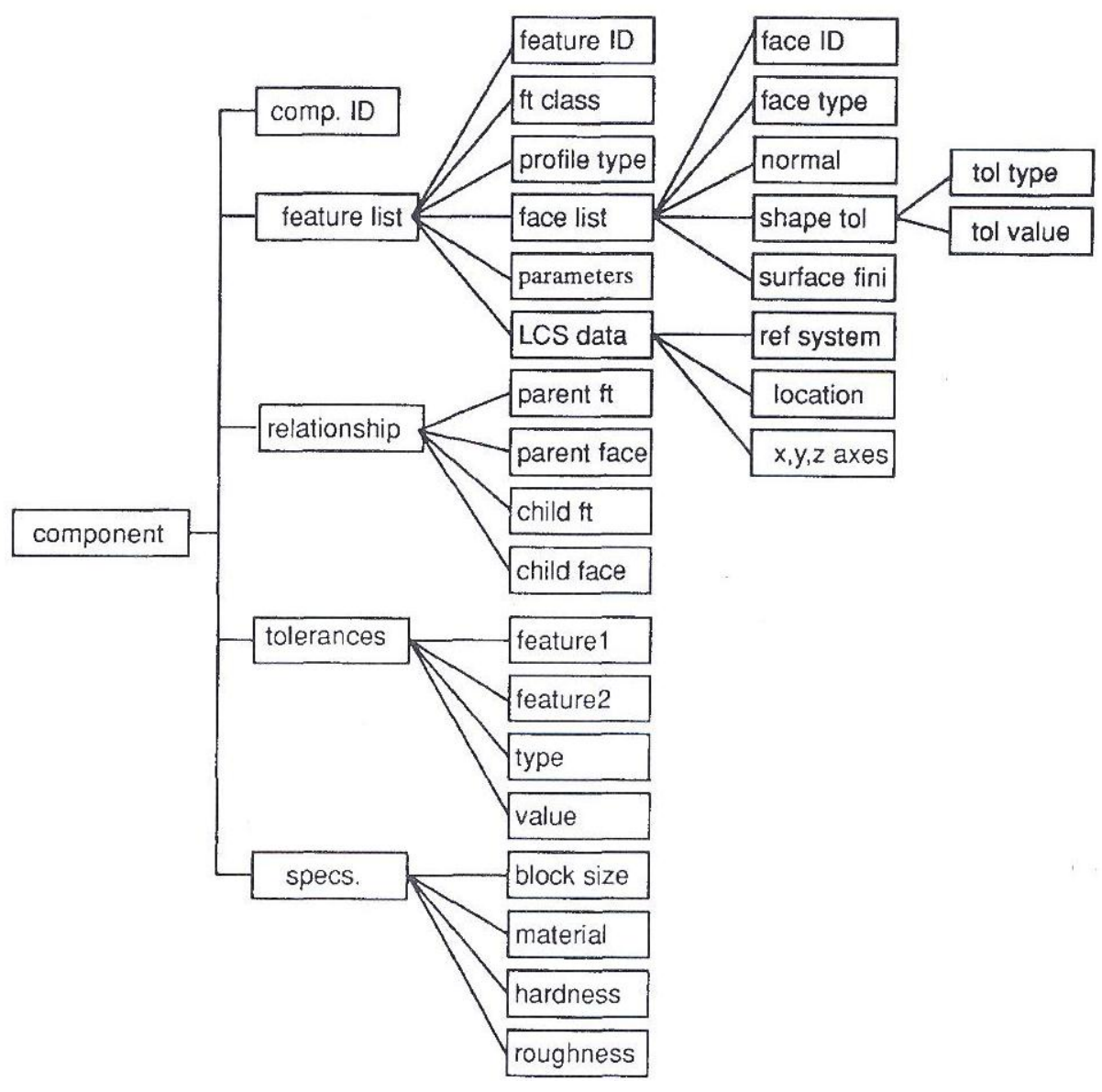

Figure 10. Component data model

each process can produce $(\mathrm{EAD}, \quad$ throughness, boundary).

A process capability model is represented using three basic elements (shape capability, technological output and optimization attributes), integrated in a common process frame. The integrated model has been illustrated on selected metal cutting processes.

Work content analysis is used for selection on machining processes at two levels: absolute and constrained knowledge levels. On the absolute level all the processes capable of transforming the required shape information into component geometry are selected, based on the process taxonomy and attached volume characteristics. At the next level the selected processes are assessed regarding their technological input and output characteristics. Based on technological constraints analysis a set of process chains is iteratively selected.

A feature-based method for component grouping has been developed. The method uses similarity between the components represented as similarity between the sets of features belonging to the components and their connectivity. Different levels of similarity have been defined in accordance with the grouping objectives which vary with different machining strategies.

Process and component grouping algorithms have been developed and the initial software implementation is currently being tested. A sample set of components has been selected from our industrial collaborators after preliminary analysis of the full production range had been carried out.

\section{Future directions and conclusions}

The design by features approach has been shown to be useful both in generating CAD models and propagating appropriate information through a manufacturing planning system for the purposes of process planning and strategic decision making in the selection of manufacturing equipment.

Proposals for future work are focused upon extending the application area of feature representations beyond manufacturing planning and into control of production. This is in recognition of the aims of concurrent engineering where there is a need to handle information relevant to every stage of a product's life-cycle. In particular, we are beginning work on the concept of 'design for production', where a highly dynamic information support system provides the designer with an indication of the effects of his decisions not only on the process capability available (process planning), but also on capacity availability (production control). We envisage a feature-based system where some of the fine detail of design is left 


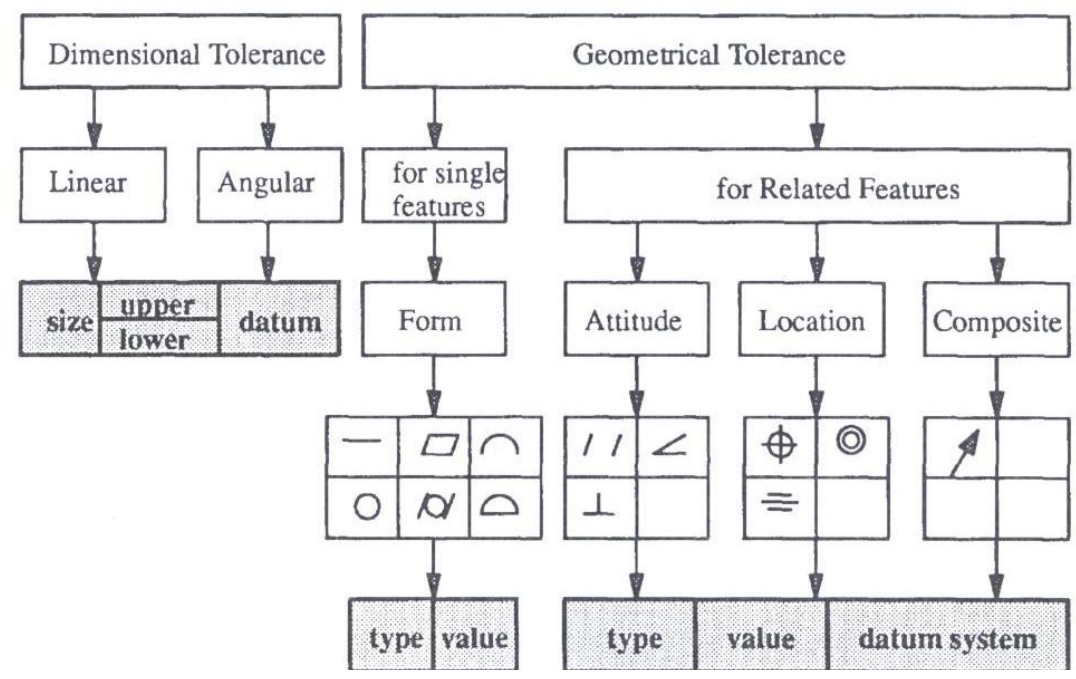

Figure 11. Tolerance annotation within LUT-FBDS

'fuzzy' so that process planning and production planning and control systems are able to reach optimum solutions without unnecessary geometric constraints. We believe that this is an essential component of highly integrated computer systems aimed at enhancing future manufacturing enterprises.

\section{Acknowledgements}

This work has been carried out under the 'Process Capability Modelling for Design and Selection of Processing Equipment' grant (GF/G35657) awarded by the Applications of Computers to Manufacturing Engineering (ACME) Directorate of the Science and Engineering Research Council (SERC). The author wishes to acknowledge his co-research workers, Dr N N Z Gindy, Dr X Gao and Dr T M Ratchev.

This paper is an adaption of a presentation to the Congresso Internacional de Computacao Grafica (CICOMGRAF 92), 30th June to 2nd July 1992, Sao Paulo, Brazil.

\section{References}

1 Gunn, T G Manufacturing for Competitive Advantage, Ballinger/Harper and Row, Cambridge, MA (1987)

2 Krause, F $\mathbf{L}$ 'Knowledge integrated product modelling for design and manufacture', Proc. 2nd Toyota Conf., Aichi, Japan (October 1988) pp 179218

3 Requicha, A A G 'Representations for rigid solids: theory, methods and systems', ACM Comput. Surv., Vol 12 No 4 (1980) pp 436-464

4 Mortenson, M E Geometric Modelling, Wiley, Chichester (1985) pp 431-437

5 Case, $\mathbf{K}$ and Gao, $\mathbf{X}$ 'Feature technology - an overview', Int. J. Comput. Integrated Manuf., Vol 6 Nos 1 \& 2 (1993) pp 2-12
6 Jared, G E M 'Reasoning and using geometric features', in J Woodwark (ed.), Geometric Reasoning, Clarendon Press, UK (1989) pp 169184

7 Herbert, P J, Hinde, C J, Bray, A D, Launders, V A, Round, $\mathbf{D}$ and Temple, $\mathbf{D}$ 'Feature recognition within a truth maintained process planning system', Int. J. Comput. Integrated Manuf., Vol 3 No 2 (1990) pp 121-132

8 Choi, B K, Barash, M M and Anderson, D C 'Automatic recognition of machined surfaces from a 3D solid model', Comput.-Aided Design, Vol16 No 2 (1984) pp 81-86

9 Mortensen, K S and Belnap, B K 'A rule-based approach employing feature recognition for engineering graphics characterisation', Comput.Aided Eng. J. (1989) pp 221-228

10 Gavankar, $\mathbf{P}$ and Henderson, M R 'Graph-based extraction of protrusions and depressions from boundary representations', Comput. Aided Design, Vol 22 (1990) pp 442-450

11 Gao, $\mathbf{X}$ and Case, $\mathbf{K}$ Process capability modelling: A review report of feature representation methodologies, Department of Manufacturing Engineering, Loughborough University of Technology (1991)

12 Cunningham, J J and Dixon, J R 'Designing with features: the origin of features', Proc. ASME Int. Comput. in Eng. Conf. and Exhibition, San Francisco, CA, Vol 1 (1988) pp 237-243

13 Pafec Limited Pafec Imaginer user Guide, Pafec Limited, Nottingham, UK (1991)

14 James, M 'Prototyping CAD', CADAM (July 1991)

15 Gindy, N N Z 'A hierarchical structure for form features', Int. J. Prod. Res., Vol 27 No 12 (1989) pp 2089-2103

16 Butterfield, W R, Green, M K, Scott, D C and Stoker, W J Part features for process planning, C- 
85-PPP-03, CAM-I Inc., Arlington, TX (1985)

17 Pratt, M J 'Applications of feature recognition in the product life-cycle', Int. J. Comput. Integrated Manuf., Vol 6 Nos 1 \& 2 (1993) pp 13-19

18 Pafec Limited Horses Kernel User Manual, Pafec Limited, Nottingham, UK (1991)

19 Spatial Technology Inc. ACIS Interface Guide, Issue 14, Spatial Technology Inc., Boulder, CO (December 1992)

20 Open Software Foundation OSF/ Motif Style Guide, Revision 1.2, Prentice-Hall, NJ (1993)

21 Case, $\mathbf{K}$ and Acar, $\mathbf{B} \mathbf{S}$ 'The manufacturing features approach to design and its impact on the learning and use of computer aided design systems', in E D Megaw (ed.), Contemporary Ergonomics, Taylor and Francis, London (1989) pp 132-137

22 Brimson, J A and Downey, $\mathbf{P} \mathbf{J}$ 'Feature technology: a key to manufacturing integration', CIM Review (Spring 1986) pp 21-27

23 ISO Industrial Automation Systems - Exchange of Product Model Data - Representation and Format Description (PDES/STEP Version 1.0), ISO Draft Proposal DP10303, National Institute of Science \& Technology (NIST), Gaithersburg, MD (1988)

24 Gindy, N N Z 'Data modelling for generative process planning', Proc. C/COMGRAF-92, Sao Paulo, Brazil (July 1992) 